\title{
Role of Deferasirox as an Adjuvant to Parenteral Antifungal Therapy in Invasive Fungal Sinusitis
}

\author{
Original Shrinivas S. Chavan', Yugandhara D. Mehere', Abhishek D. Khond ${ }^{1}$ and \\ Article \\ Rahul Radhakrishnan ${ }^{2}$
}

${ }^{1}$ Department of Otorhinolaryngology, ${ }^{2}$ Department of General Medicine, Grant Government

Medical College and Sir J.J. Group of Hospitals, Mumbai, India

\begin{abstract}
Background: Invasive Fungal Sinusitis(IFS) is seen in both immunocompromised and immunocompetent patients. All fungi elaborate uptake mechanisms to sequester iron, and produce siderophores with higher affinity for iron. The availability of iron and ability to utilize them are essential for viability and growth of Mucor and Aspergillus. Currently, IFS is dealt with surgical debridement and aggressive medical therapy. Amphotericin B being currently used in medical management. Considering the importance of iron in fungal growth, addition of iron chelating agent as an adjunct has potential beneficial role. The newer iron chelator deferasirox, share higher affinity for iron and hence, deprive the fungi of iron, inhibiting their growth. However, the evidence for deferasirox as adjunct therapy in IFS is limited thus, prompting us for this study.

Aim: To study the role of deferasirox as an adjuvant to parenteral antifungal therapy in invasive fungal sinusitis.

Materials and Methods: A prospective interventional comparative clinical study was conducted. A total of 42 IFS patients were selected for study. About 12 patients were lost due to attrition. Remainder of 30 patients were evenly randomized into Group A and B by chit allocation. After surgical debridement, Patients in group A received only parenteral Amphotericin B and group B received parenteral Amphotericin B and oral deferasirox. The outcomes in two groups were compared clinically,endoscopically and radiologically and analysed using Mann Whitney test.

Conclusion: Parenteral Amphotericin B and deferasirox combination is better than parenteral Amphotericin B alone in treatment of IFS.
\end{abstract}

Key Words: Amphotericin B, deferasirox, invasive fungal sinusitis, iron chelators.

Received: 05 April 2021, Accepted: 14 May 2021

Corresponding Author: Abhishek D. Khond, Department Of Ent, Grant Governement Medical College and Sir J.J. Group Of Hospitals, Byculla ,Mumbai. Maharashtra University of Health Sciences, Nashik, Maharashtra, India, Tel.: +919867662004, E-mail: abhishek.khond@yahoo.com

ISSN: 2090-0740, 2021

\section{INTRODUCTION}

Invasive Fungal Sinusitis affects both
immunocompromised patients as well as
immunocompetent patients. But outcomes are more
catastrophic in immunocompromised patients. In $80 \%$ of
cases, fungi responsible belongs to order Zygomycetes,
which involves mucor, rhizopus, rhizomucor, and
absidia ${ }^{[1]}$. Its subgroup, mucormycosis is a life-threatening
infection caused by fungi of the order Mucorales $^{[1]}$. In
remaining cases aspergillus fumigatous is predominant
fungal pathogen $^{[1]}$.

All fungi elaborate specific uptake mechanisms to sequester iron, and most commonly produce small molecules with high affinity for iron,the siderophores. The importance of iron appears to be particularly high for mucorales, which grow abundantly in iron-rich media. In addition all the known predisposing factors for Mucorales have, as a common feature, the increased availability of free iron ${ }^{[2]}$. The availability of iron and the ability to take up and utilize available iron are also essential for the viability of other fungi like Aspergillus fumigatus ${ }^{[3]}$. In todays era, invasive fungal sinusitis is dealt with radical surgical debridement and aggressive medical therapy ${ }^{[4]}$. At present amphotericin B continues to be current standard of care in medical management of mucormycosis ${ }^{[4]}$. Considering the importance of iron in fungal growth, addition of iron chelating agent as an adjunct has potential beneficial role. The iron chelator, deferoxamine acts as xenosiderophore, and supports the growth of Zygomycetes ${ }^{[2]}$. Conversely, the newer iron chelators deferasirox and deferiprone, share higher affinity constants for iron and, as a result, deprive the fungi of iron, inhibiting their growth ${ }^{[2]}$. However, the evidence for deferasirox as adjunct thearpy in mucormycosis is very limited thus, prompting us to undertake this study.

In this study, we evaluated the efficacy of deferasirox as an adjuvant to parenteral antifungal therapy in invasive fungal sinusitis, in terms of clinical and radiological improvement. 


\section{MATERIALS AND METHODS}

\section{STUDY DESIGN}

This was a prospective interventional and comparative study conducted between December 2018 to December 2020 in Depart $\neg$ ment of Otorhinolaryngology, Grant Government Medical College and Sir J.J. Hospital ,mumbai India. Patients attending ENT OPD with symptoms suggestive of persistent nasal blockade, nasal discharge , epistaxis, blurring of vision, orbital swelling, proptosis, ptosis, opthalmoplegia, facial nerve paralysis, facial pain ,cheek swelling, were screened and subjected to anterior rhinoscopy and computed tomography of nose and paranasal sinuses, orbit and brain depending on symptomatology. Further, nasal endoscopic biopsy was taken. The biopsy specimen was sent for histopathological examination and fungal culture. Histopathologically proven cases of invasive fungal sinusitis were segregated and 42 such patients were selected for this study. But rhinocerebral mucormycosis being life threatening disease 4 patients succumbed to the disease before any intervention. Further 8 patients lost follow up during the course of treatment. Remainder of 30 patients were then enrolled in the study after taking an informed consent and acquiring the ethical committee clearance.

\section{Inclusion criteria}

1. Histopathologically proven cases of invasive fungal sinusitis

2. Patients of all age groups and sex.

3. Patients willing to participate in the study.

\section{Exclusion criteria}

1. Patients not willing to participate in the study

2. No evidence of invasive fungal sinusitis on histopathology or fungal culture.

3. Renal failure, hepatic insufficiency.

All patients were subjected to a questionnaire for calculating the symptom score by using Sinonasal Outcome Test (SNOT-22 SCORE) ${ }^{[5]}$.

All 30 Biopsy proven cases of invasive fungal sinusitis were subjected to radical surgical debridement under appropriate anaesthesia. All patients were operated by the same surgeon who was blinded with respect to study designs and study details. After surgical debridement these Patients were randomized into Group A and Group B by chit allocation technique, 15 patients in each group. Patients in group A received only parenteral Amphotericin B $(>5 \mathrm{mg} / \mathrm{kg}$ ) and in group B received parenteral Amphotericin B ( $>5 \mathrm{mg} / \mathrm{kg})$ and oral Deferasirox (20 mg $/ \mathrm{kg} /$ day). All patients in group A and group B were followed up at, 1 month, 3 months and 6 months postoperatively with same postoperative care. On each follow up day patients were subjected to a similar questionnaire which was given pre operatively to calculate the symptom score. General clinical examination, anterior rhinoscopy, and nasal endoscopy were performed on each follow up day. Endoscopic findings were graded by using Lund Kennedy Endoscopic Scoring System ${ }^{[6]}$.

Post operatively radiological assessment was done in the form of CT PNS during $6^{\text {th }}$ months follow up and Lund Mackay Score was applied for analysing the results.

The Lund-Mackay score ${ }^{[7]}$ is a widely used method for radiologic staging of chronic rhinosinusitis. Each side is graded separately. A combined score of up to 24 is possible.

Symptoms score, computed tomgraphic score and endoscopic score were compared pre-operatively and post-operatively between group A and group B. Possible complications arising in both the groups were documented on aforementi-oned days. Mortalities occurring in both groups were documented.

\section{DATA ANALYSIS AND STATISTICAL TESTS}

All data collected were entered in Microsoft Excel sheet and transferred to SPSS ver. 17 software for statistical analysis. Quantitative data was presented as mean and standard deviation and comparison of the two study groups was done using Mann Whitney test. Pre-operative and post -operative quantitative data of each group was compared using Mann Whitney test. Qualitative data was presented as frequency and percentage and analysed using Mann Whitney test. A $p$-value of $<0.05$ was considered as statistically significant.

\section{RESULTS}

In this study 30 patients were assessed with total study duration of 18 months. Each group consisted of 15 patients.

The age group of the patients ranged from 27- 70 yrs and the mean age of the patients in the Amphotericin study group was $53.66 \pm 13.70$ and in the Amphotericin and Deferasirox group was $50.53 \pm 10.03$ (Table 1).

The mean age of both groups were comparable and statistically insignificant.

Table 1: Demographics of Group A And Group B

\begin{tabular}{ccc}
\hline & Group A & Group B \\
\hline Mean Age & $53.66(13.70)$ & $50.53(10.03)$ \\
& P Value $=0.394$ (Mann Whitney) & \\
Gender & & \\
Male & 11 & 10 \\
Female & 4 & 5 \\
\hline
\end{tabular}

In our study majority of patients were male (70\%). In group A male:female ratio was 2.75:1 and in Group B. it was $2: 1$. The gender distribution in both groups were comparable and statistically insignificant. 


\section{SNOT-22 SYMPTOM SCORE}

Table 2: Mean Snot- 22 During Pre And Post Intervention In Both Groups

\begin{tabular}{cccc}
\hline Mean Snot & Group A & Group B & $\begin{array}{c}\text { P Value } \\
\text { (Mann Whitney) }\end{array}$ \\
\hline Pre & $70.60(16.86)$ & $65.40(18.10)$ & 0.394 \\
1 Month Post & $37.60(9.60)$ & $32.40(9.37)$ & 0.162 \\
3 Month Post & $31.33(8.33)$ & $28.80(10.18)$ & 0.555 \\
& $15.26(4.65)$ & $10.53(3.58)$ & 0.005 \\
6 Month Post & $<0.01$ & $<0.01$ & \\
\hline
\end{tabular}

When mean SNOT-22 score pre intervention was compared between two groups, it was found to be comparable and statistically insignificant. Although there was statistically significant improvement in SNOT-22 in both groups when comparison was done between pre and 6 months follow up $(p<0.01)$. But after intervention when both groups were compared with each other, the results were found to be statistically insignificant during 1 month and three month follow up but on 6 th month follow up there was significant difference between two groups $(p=0.005)$ which showed better improvement in symptom SNOT-22 score in the Group B (Table 2).

\section{LUND KENNEDY ENDOSCOPIC SCORE}

Table 3: Mean Endoscopic Score During Pre And Post Intervention In Both Groups

\begin{tabular}{cccc}
\hline $\begin{array}{c}\text { Endoscopic } \\
\text { Score }\end{array}$ & Group A & Group B & $\begin{array}{c}\text { P Value } \\
\text { (Mann Whitney) }\end{array}$ \\
\hline Pre & $12.93(2.54)$ & $13.13(1.84)$ & 0.967 \\
1 Month Post & $8.40(2.02)$ & $8.13(1.99)$ & 0.896 \\
3 Month Post & $\begin{array}{c}4.12(1.06) \\
\text { 3 Month Post }\end{array}$ & $\begin{array}{c}1.53(0.63) \\
<0.001\end{array}$ & $\begin{array}{c}0.33(0.79) \\
<0.001\end{array}$ \\
\hline
\end{tabular}

When mean endoscopic score pre intervention was compared between two groups, it was found to be comparable and statistically insignificant (Table 3). Although there was statistically significant improvement in mean endoscopic score in both groups when comparison was done between pre and 6 month follow up $(p<0.001)$. But after intervention when both groups were compared with each other, the results were found to be statistically insignificant during 1 month and three month follow up but on 6 th month follow up there was significant difference between two groups $(p=0.029)$ which showed better improvement in symptoms of mean endoscopic score in Group B. (Figure 1)

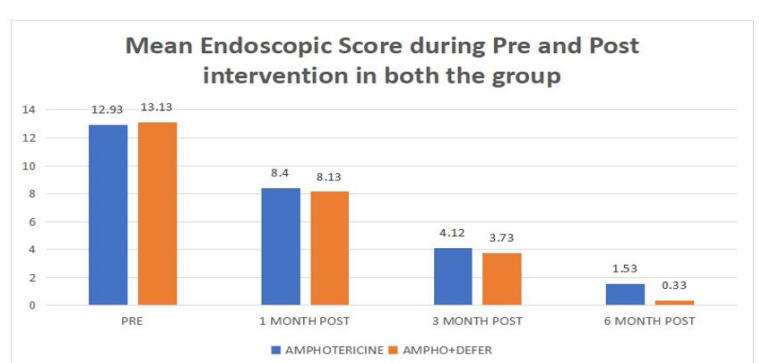

Fig. 1: Mean endoscopic score during pre and post intervention in both groups

\section{LUND MACKEY RADIOLOGICAL SCORE}

Table 4: Mean Ct Score During Pre And Post Intervention In Both Groups

\begin{tabular}{cccc}
\hline Ct Score & Group A & Group B & $\begin{array}{c}\text { P Value } \\
\text { (Mann Whitney) }\end{array}$ \\
\hline Pre & $\begin{array}{c}14.66(3.59) \\
\text { 6 Month Post }\end{array}$ & $\begin{array}{c}4.90(3.01) \\
<0.001\end{array}$ & $\begin{array}{c}3.20(1.17) \\
<0.001\end{array}$ \\
\hline
\end{tabular}

When mean CT score pre intervention was compared between two groups, it was found to be comparable and statistically insignificant (Table 4). Although there was statistically significant improvement in mean CT score in both groups when comparison was done between pre and 6 month follow up $(\mathrm{p}<0.01)$. But on 6 th month follow up there was significant difference between two groups $(p=0.025)$ which showed better improvement in radiological score in Group B. (Figure 2)

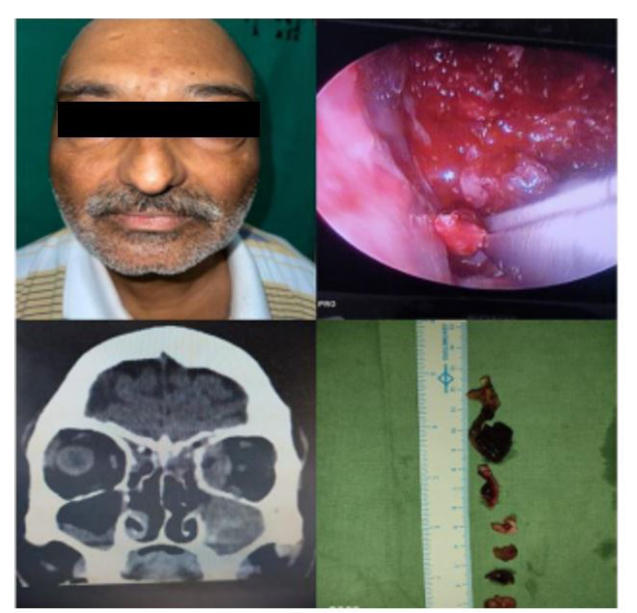

Fig. 2: A case of mucormycosis successfully treated with surgical debridement followed by medical management in the form of Amphotericin B and Deferasirox 


\section{MEAN BLOOD SUGAR LEVELS}

Table 5: Mean Blood Sugar During Pre And Post Intervention In Both Groups

\begin{tabular}{cccc}
\hline Blood Sugar & Group A & Group B & $\begin{array}{c}\text { P Value } \\
\text { (Mann Whitney) }\end{array}$ \\
\hline Pre & $\begin{array}{c}364.66 \\
(144.11)\end{array}$ & $\begin{array}{c}420.33 \\
(92.70)\end{array}$ & 0.299 \\
& 244.13 & 230.86 & 0.740 \\
Post & $(108.72)$ & $(112.23)$ & \\
$\begin{array}{c}\text { P Value } \\
\text { (Wilcoxan Test) }\end{array}$ & 0.0001 & 0.0001 & \\
\hline
\end{tabular}

The mean blood sugar levels in both groups preoperatively were comparable and statistically insignificant. But when compared pre and post operatively,

It showed statistically significant improvement in the blood sugar levels in both groups equally (Table 5).

\section{SAFETY ANALYSIS}

On comparing two groups after starting medical line of management in the form of Amphotericin B and Amphotericin B + Deferasirox , there was no statistical difference between two groups in terms of new onset, serious adverse events between two groups.

\section{DISCUSSION}

This study was conducted for analysing the role of deferasirox as an adjunct to parenteral antifungal therapy for Invasive fungal sinusitis. Invasive fungal sinusitis is manifested as variety of different syndromes in humans such as rhino-orbito-cerebral syndrome, pulmonary syndrome and other disseminated forms particularly in immunocompromised and diabetic patients. Invasive fungal sinusitis are broadly of two types i.e mucormycosis and Invasive Aspergillosis ${ }^{[8]}$. Mucor, Rhizopus, rhizomucor and absidia are causative agents seen in mucormycosis and aspergillus fumigatous and A. flavus are main pathogens causing invasive aspergillosis ${ }^{[8]}$. The management of invasive fungal sinusitis includes surgical debridement and parenteral antifungal therapy. Currently, the first line antifungal therapy in use is parenteral amphotericin B, preferably liposomal due to less nephrotoxicity ${ }^{[8]}$. Considering the importance of iron in fungal growth, addition of iron chelating agent as an adjunct has potential beneficial role.The newer iron chelators deferasirox and deferiprone, share higher affinity constants for iron and, as a result, deprive the fungi of iron, inhibiting their growth ${ }^{[2]}$. However, the evidence for deferasirox as adjunct thearpy in mucormycosis is very limited thus, prompting us to undertake this study.

In this study we evaluated 30 patients of histopathologically proven invasive fungal sinusitis. All of them underwent radical surgical debridement in the form of endoscopic sinus surgery for decreasing the fungal load in the patient. Further these patients were randomly divided into two groups, group A recieved parenteral
Amphotericin B only and group B recieved combination of parenteral Amphotericin B and oral Deferasirox. The two groups were compared preoperatively and postoperatively on basis of improvement of symptoms score, control of blood sugar levels and control of disease endoscopically and radiologically .

In our study, the average age of patients ranged from 27- 70yrs and the mean age of the patients in Group A was $53.66 \pm 13.70$ and in Group B was $50.53 \pm 10.03$. Both groups were comparable and their difference was statistically insignificant. These results were comparble to study by Spellberg et al. where mean age was $53^{[9]}$. As type 2 diabetes mellitus is more common in this age group, this might be probable cause for higher incidences of invasive fungal sinusitis in them.

In this study majority of patients were male (70\%). In group A male: female ratio was 2.75:1 and in group $B$ it was 2:1. The gender distribution in both groups were comparable and their difference was statistically insignificant. Our result was comparable to Gillespie et al. Where male: female ratio is $2.12: 1^{[4]}$. As diabetes is more common in male, and as male seems more susceptible than female to consequences of indolence and obesity, possibly due to difference in insulin sensitivity and regional fat deposition which lead to preponderance of more cases of invasive fungal sinusitis in male.

In this study, when mean symptom score (SNOT-22) pre intervention was compared between two groups, difference was found statistically insignificant. But after intervention when both groups were compared with each other, the results were found to be statistically insignificant during one and three month follow up but on $6^{\text {th }}$ month follow up there was significant difference between two groups $(p=0.005)$ which showed better improvement in symptom SNOT-22 score in the Group B.

On admission mean endoscopic score pre-intervention was compared between two groups, and was statistically insignificant. But after intervention when both groups were compared with each other, the results were found to be statistically insignificant during 1 month and three month follow up but on $6^{\text {th }}$ month follow up there was significant difference between two groups $(p=0.029)$ which showed better improvement in mean endoscopic score in Group B.

Similarly, when mean pre-intervention CT score was compared between two groups, it was found to be comparable and statistically insignificant. But, on $6^{\text {th }}$ month follow up there was significant difference between two groups ( $p=0.025$ ) which showed better improvement in radiological score in Group B.

Similar study conducted by Onal et al, which compared radiological improvement in a 69 years old diabetic patient with mucormycosis which was succesfully treated with liposomal amphotericin B (LAmB), posaconazole and deferasirox. In this study, there was no improvement noted, following treatment with LAmB and Posaconazole. 
Thereafter, Deferasirox was added on $15^{\text {th }}$ day of treatment for two weeks period. Following which, MRI results showed improvement when compared to previous MRI ${ }^{[8]}$.

Similarly, Reed et al., also compared radiological improvement in a patient with progressive rhinocerebral mucormycosis with orbital exenteration and completion of LAmB and deferasirox therapy ${ }^{[10]}$.

Ibrahim et al. reported that 28 of 29 clinical Mucorales isolates were inhibited in vitro by the deferasirox iron chelation and when this iron chelator, deferasirox, was administered to diabetic ketoacidotic or neutropenic mice, a significant improvement of survival rates and host inflammatory response plus reduced fungal tissue burden with combination of LAmB were observed. In DKA mice with disseminated mucormycosis, deferasirox was as effective as LAmB therapy, and combination deferasiroxLAmB therapy synergistically improved survival $(80 \%$ survival for combination vs. $40 \%$ for monotherapy vs. $0 \%$ for placebo). In particular, combination therapy resulted in a 100 -fold decrease in brain fungal burden, compared with monotherapy and improved survival ${ }^{[11]}$. These data are concordant with our study in humans.

The results of our study was seen contradicting to previous study conducted by Spellberg et al., which compared $\mathrm{LAmB}+$ deferasirox with $\mathrm{LAmB}+$ placebo in a small randomized controlled trial which showed no clinical improvement in patients on deferasirox. There are several possible explanations for discrepancy between the promising efficacy of defarisirox seen in other studies and results observed in this study. First, despite its fungicidal effects on Mucorales in vitro and substantial efficacy in mice, deferasirox may have no impact on clinical disease or may even worsen the clinical course of mucormycosis in humans. There are no published reports of deferasirox antagonism with other antifungal agents and the drug does not promote fungal growth in vitro or in mice. Hence, mechanisms by which the drug could worsen clinical infection are not clear. Second, efficacy of deferasirox was most apparent in mice with DKA, which have increased free iron levels, and efficacy was substantially less apparent in neutropenic mice ${ }^{[9]}$.

Although there was no apparent difference in the number or type of non SAEs by 180 days of follow up, the mortality rate was higher in Amphotericin B plain than those treated with Amphotericin B with deferasirox. The primary driver of excess mortality appeared to be clinical failure due to progression of disease rather than any specific to toxicity. The possible utility of oral iron chelating agent, deferasirox, as an adjunctive therapy for mucormycosis have been evaluated in small studies with confliction results.However, in clinical practice there are success and failure reports related to deferasirox in mucormycosis.

\section{CONCLUSION}

To conclude Amphotericin B and Deferasirox combination is better than parenteral Amphotericin B alone in treatment of invasive fungal sinusitis when the effects were compared clinically, endoscopically and radiologically. In our study Deferasirox was found to be relatively well tolerated and safe with statistically significant improvement in patients condition where the combination of Amphotericin B and Deferasirox was used after surgical debridement.

When compared with previous studies, there was discrepancy between the results, with some studies showing improvement in the patients condition while other studies showing no statistically significant difference or even worsening of the patients condition. For this reason, a more elaborate and larger randomised study for finding out the role of deferasirox in invasive fungal sinusitis will definitely be helpful to confirm or refute our results.

\section{CONFLICT OF INTERESTS}

There are no conflicts of interest.

\section{REFERENCE}

1. Spellberg B, Edwards J, Ibrahim A. Novel perspectives on mucormycosis: pathophysiology, presentation, and management. Clinical microbiology reviews. 2005 Jul 1;18(3):556-69.

2. Symeonidis AS. The role of iron and iron chelators in zygomycosis. Clinical Microbiology and Infection. 2009 Oct;15:26-32Hibbett DS, Binder M, Bischoff JF, Blackwell M, Cannon PF, Eriksson OE, Huhndorf S, James T, Kirk PM, Lücking R, Lumbsch HT. A higher-level phylogenetic classification of the Fungi. Mycological research. 2007 May 1;111(5):509-47.

3. Nazik H, Penner JC, Ferreira JA, Haagensen JA, Cohen K, Spormann AM, Martinez M, Chen V, Hsu JL, Clemons KV, Stevens DA. Effects of iron chelators on the formation and development of Aspergillus fumigatus biofilm. Antimicrobial agents and chemotherapy. 2015 Oct 1;59(10):6514-20.

4. Gillespie MB, O'Malley BW, Francis HW. An approach to fulminant invasive fungal rhinosinusitis in the immunocompromised host. Archives of Otolaryngology-Head \& Neck Surgery. 1998 May 1;124(5):520-6.

5. Drahaman AP, Henney SE. Using SNOT-22 as a decision making tool in performing Nasal Surgery. Ceylon Journal of Otolaryngology. 2015 Nov 23;4(1).

6. Psaltis AJ, Li G, Vaezeafshar R, Cho KS, Hwang $\mathrm{PH}$. Modification of the Lund-Kennedy endoscopic scoring system improves its reliability and correlation with patient-reported outcome measures. The Laryngoscope. 2014 Oct;124(10):2216-23.

7. Okushi T, Nakayama T, Morimoto S, Arai C, Omura K, Asaka D, Matsuwaki Y, Yoshikawa M, Moriyama H, Otori N. A modified Lund-Mackay system for radiological evaluation of chronic rhinosinusitis. Auris Nasus Larynx. 2013 Dec 1;40(6):548-53. 
8. Önal U, Taşbakan M, Yamazhan T, Arda B, Pullukçu H, Ulusoy S, Sipahi OR. Treatment of Mucormycosis with Liposomal Amphotericin B, Posaconazole and Deferasirox: A Case Report. Journal of Microbiology \& Infectious Diseases. 2016 Mar 1;6(1).

9. Spellberg B, Ibrahim AS, Chin-Hong PV, Kontoyiannis DP, Morris MI, Perfect JR, Fredricks D, Brass EP. The Deferasirox-AmBisome Therapy for Mucormycosis (DEFEAT Mucor) study: a randomized, double-blinded, placebo-controlled trial. Journal of Antimicrobial Chemotherapy. 2012 Mar 1;67(3):715-22.
10. Reed C, Ibrahim A, Edwards JE, Walot I, Spellberg B. Deferasirox, an iron-chelating agent, as salvage therapy for rhinocerebral mucormycosis. Antimicrobial agents and chemotherapy. 2006 Nov 1;50(11):3968-9.

11. Ibrahim AS, Gebermariam T, Fu Y, Lin L, Husseiny MI, French SW, Schwartz J, Skory CD, Edwards JE, Spellberg BJ. The iron chelator deferasirox protects mice from mucormycosis through iron starvation. The Journal of clinical investigation. 2007 Sep 4;117(9):2649-57. 